\title{
Obesity and Diabetes, (Diabesity) risk factors
}

\author{
Khalid Khamees H ${ }^{1}$, Mohamed Osman Elamin ${ }^{1,}{ }^{*}$, Meshari F Saleh A ${ }^{2}$ and Mohamed H Alfahmi ${ }^{3}$ \\ ${ }^{1}$ Faculty of Public Health \& Informatics, Umm Al-Qura University. \\ ${ }^{2}$ King Abdullah Medical City. KSA. \\ ${ }^{3}$ Ministry of Health. KSA.
}

International Journal of Science and Research Archive, 2022, 05(01), 001-008

Publication history: Received on 01 December 2021; revised on 05 January 2022; accepted on 07 January 2022

Article DOI: https://doi.org/10.30574/ijsra.2022.5.1.0208

\begin{abstract}
Introduction: Glucose intolerance, including overt diabetes, was associated with obesity in BMI from $\geq 25 a n d$ was $85.7 \%$ of cases in recent studies. The reasons are not entirely clear, but studies have shown that there is a significant association between diabetes and obesity for several factors, including lifestyle, genetic factors and others.
\end{abstract}

Objective: The purpose of the review of these studies is to clarify the relation of diabetes, obesity, risk factors affecting them and the importance of prevention.

Methods:An electronic search of the PubMed database was performed to obtain key literature in the field of obesity and its association with diabetes.

Results: The review clarified that around one third of Saudi patients are diabetics, in addition to that $85 \%$ of them were overweight or obese.

Discussion: It has been clarified that there are strong relations of obesity and Diabetes, both are interconnected, Obesity is a major risk factor for Diabetes.

Conclusion: The review concluded that there were strong relations between obesity and diabetes, although there are multiple factors associated with diabetes, but obesity constitutes the major risk factor. The review recommends extensive health education and promotion programs to be initiated to raise awareness on obesity and diabetes relations and possible prevention measures.

Keywords: Obesity; Diabetes; Diabesity; Risk; Factors

\section{Background}

Non-communicable diseases (NCDs), also known as chronic diseases, tend to be of long duration and are the result of a combination of genetic, physiological, environmental and behavioral factors. The main types of NCDs are cardiovascular diseases (like heart attacks and stroke), cancers, chronic respiratory diseases (such as chronic obstructive pulmonary disease and asthma) and diabetes.NCDs disproportionately affect people in low- and middle-income countries where more than three quarters of global NCD deaths - 31 million - occur. ${ }^{1}$ apnea, hyperlipidemia, and osteoarthritis.

\footnotetext{
${ }^{*}$ Corresponding author: Dr. Mohamed Osman Elamin

Faculty of Public Health, Umm Al-Qura University, Makkah, KSA.

Copyright (C) 2022 Author(s) retain the copyright of this article. This article is published under the terms of the Creative Commons Attribution Liscense 4.0.
} 


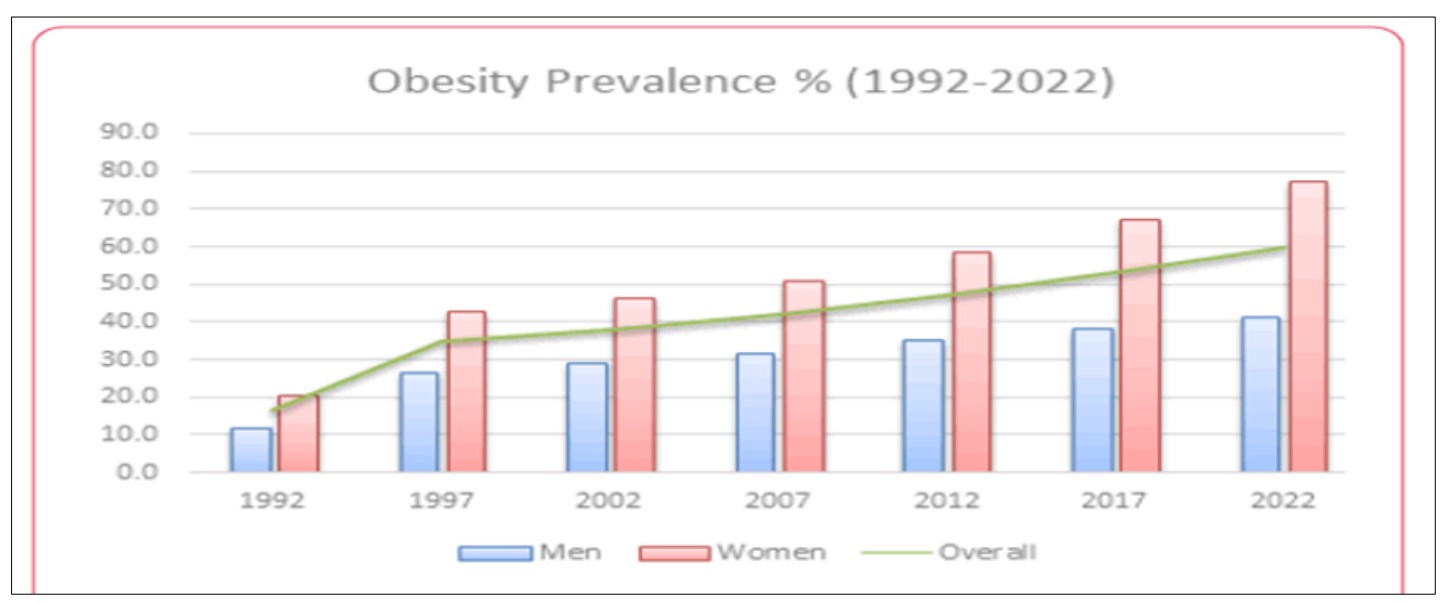

Figure 1 Obeity prevalence \% (1992-2022). As indicated earlier, the current research paper adopts a qualitative approach and follows a review- design to explore the research problem; i.e. to examine the prevalence of obesity in KSA, and explain its causes and consequences, the study performed a detailed literature review. The following table presents a summary of the reviewed studies in order to highlight and discuss the key statistics and findings. Data Source: [18]

\subsection{Diabetes in global situation}

Diabetes Mellitus (DM) is one of the fastest-growing health problem in the world, which is now reaching to epidemic proportion in some countries. It is mainly due to consequence of life-style as lack of exercise, unhealthy diet, obesity and overweight. Over the past four decades major socio-economic changes have occurred in Saudi Arabia.The growth and prosperity have brought pronounced changes in the lifestyle of the people. Most notably, eating habits are less healthful and the level of physical activity has declined. There is increased consumption of fast foods and sugar-dense beverages (e.g., sodas). Simultaneously, technological advances - cars, elevators, escalators, remotes - have led to a decrease in level of activity. Traditional dependence on locally grown natural produce such as fruits, vegetables and wheat has also shifted. This has resulted in the dramatic increase in the diabetes prevalence. ${ }^{2}$

Globally in 2013, it is estimated that almost 382 million people suffer from diabetes with a prevalence of $8.3 \%$. Top 10 countries with higher prevalence of diabetes are Tokelau (37.5\%), Federated States of Micronesia (35\%), Marshall Islands (34.9\%), Kiribati (28.8\%), Cook Islands (25.7\%), Vanuatu (24\%), Saudi Arabia (23.9\%), Nauru (23.3\%), Kuwait (23.1\%) and Qatar (22.9\%). So Saudi Arabia is among top ten countries of the world with highest prevalence. ${ }^{3}$

\subsection{Diabetes in (KSA)}

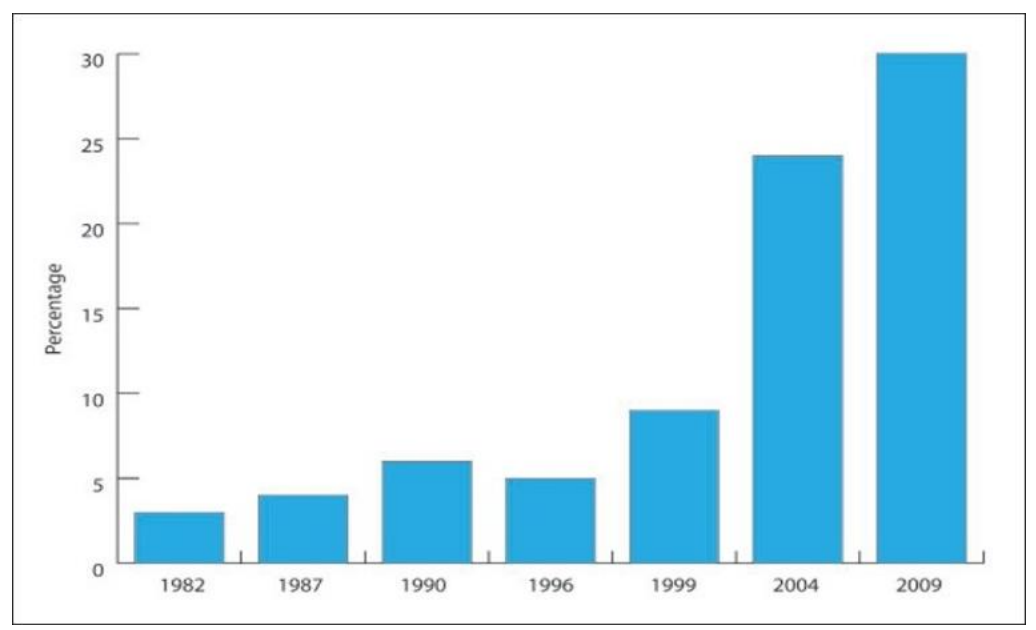

Figure 2 Epidemiological studies of the prevalence of diabetes in Saudi Arabia, 1982-2009. ${ }^{8}$

A community-based national epidemiological health survey was conducted among Saudi subjects in the age group of 30-70-years of selected households over a 5-year period. Data were obtained from history, fasting plasma glucose levels, and body mass index. The data were analyzed to classify individuals as diabetic, impaired fasting glucose and 
normal. A total of 17232 Saudi subjects were selected in the study, and 16917 participated ( $98.2 \%$ response rate). Four thousand and four subjects (23.7\%), out of 16917 were diagnosed to have DM. Thus, the overall prevalence of DM obtained from this study is $23.7 \%$ in KSA.

Diabetes mellitus was more prevalent among Saudis living in urban areas than rural and male study participants. ${ }^{4,5}$

\subsection{Relation between obesity and diabetes}

Obesity is a disorder caused by an imbalance between the energy consumed as food (calories) and the energy used by our body (during physical activities and regular functioning of the body). Obesity occurs mainly due to changes in dietary habits and lifestyle. Body Mass Index (BMI) is an indicator of obesity (Figure4). Developments in technology, while improving productivity, have on the whole, driven individuals towards a sedentary lifestyle.

Furthermore, physical activity has been limited largely to sports and gyms, whereas in the past it was integrated into our day to day activities. It should also be mentioned that our eating style has moved towards quicker preparations and unhealthier, processed options, often high in sugar. So what does this mean for us? Unhealthy food habits which involve an excessive consumption of foods rich in sugar lead to excess energy in the body. This unused energy is deposited as fats and leads to increased sugar levels in our blood. While our body is designed to process high sugar loads in the short term, over a longer time frame, sugar in the bloodstream is not adequately taken up by the body and this leads to insulin resistance. ${ }^{17}$ Insulin resistance is the common underling cause for both Type 2 Diabetes and obesity. ${ }^{6,7}$

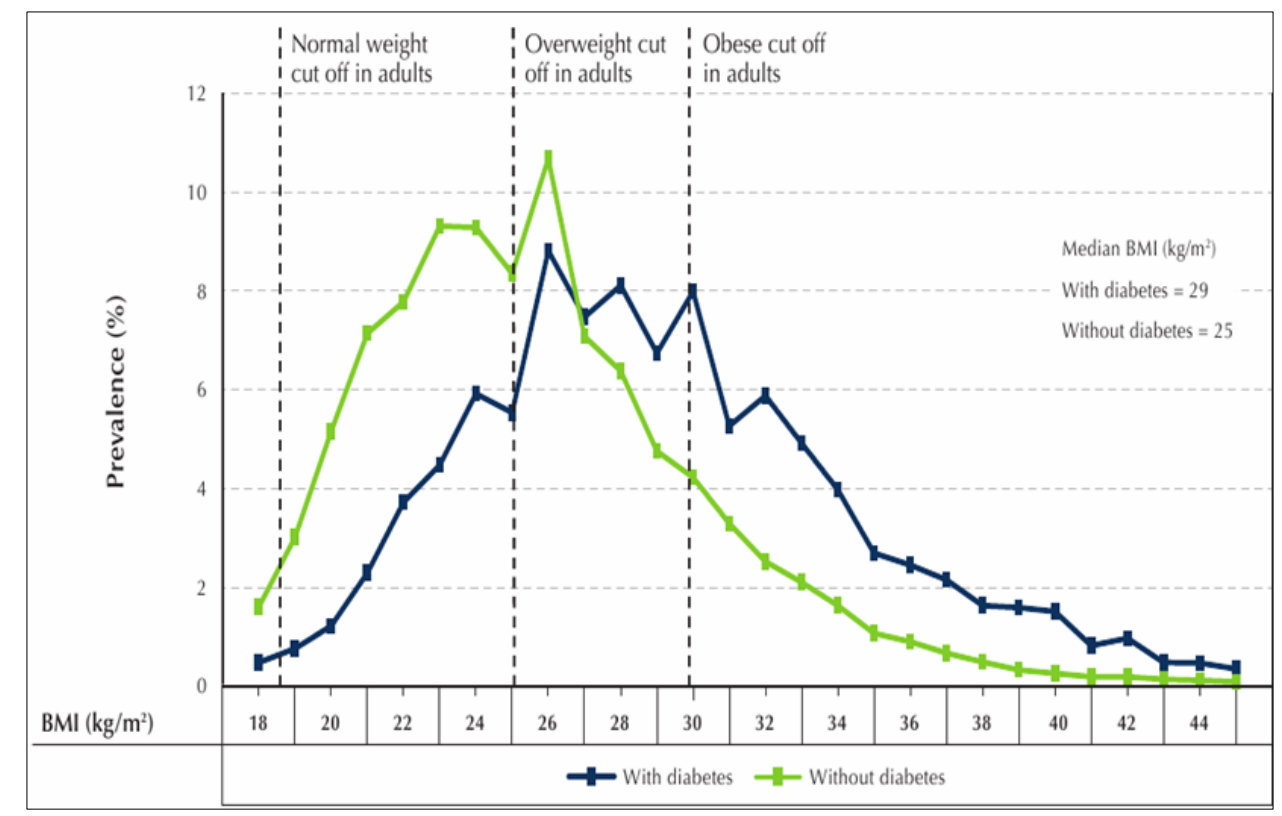

Figure 3 Distribution of self-reported body mass index (BMI) among individuals aged 18 years and older, by diabetes status

\section{Material and methods}

An electronic search of the PubMed database was performed to obtain key literature in the field of obesity and its association with diabetes using the following search terms: <obesity>, <diabetes> and <Diabesity>. The PubMed database was chosen as it remains the most widely used resource of medical journals and indexes only peer-reviewed literature.

\section{Results}

A total of 6024 patients attending the Department of Primary Care were included in the study of (Prevalence of diabetes mellitus in a Saudi community),there were 2279 (37.8\%) males and 3744 (62.2\%) females (Table1). Diabetes was present in 1792 (30\%) patients. Males were older than females (57.5 [13.1] vs. 53.7 [13.1] years, respectively, $P<.0001$ ). 
Table 1 Characteristics of patients by disease status

\begin{tabular}{|l|l|l|}
\hline Parameter & Diabetes & No diabetes \\
\hline Gender & & \\
\hline Male & $768(34.1)$ & $1486(65.9)$ \\
\hline Female & $1024(27.6)$ & $2690(72.4)$ \\
\hline Age(years) & $55.3(13.2)$ & $36.0(14.8)$ \\
\hline Age group (years) & & \\
\hline $12-19$ & $8(2)$ & $400(98)$ \\
\hline $20-29$ & $61(4.6)$ & $1265(95.4)$ \\
\hline $30-39$ & $147(12.1)$ & $1070(87.9)$ \\
\hline $40-49$ & $326(31.9)$ & $696(68.1)$ \\
\hline $50-59$ & $558(58.2)$ & $401(41.8)$ \\
\hline $60-69$ & $447(68.6)$ & $205(31.4)$ \\
\hline$\geq 70$ & $245(63.8)$ & $139(36.2)$ \\
\hline
\end{tabular}

The prevalence of diabetes was $34.1 \%$ in males and $27.6 \%$ in females $(P<.0001)$. Comparing the prevalence by gender in different age groups showed that females younger than $<50$ years had a higher prevalence than males in the corresponding age range, $34.1 \%$ vs. $25.1 \%$, respectively $(P<.0001)$. In males older than 50 years, the prevalence was higher when compared with females in a similar age range (Table 2). The prevalence of body mass index (BMI) of $\geq 25$ was $72.5 \%$.

Table 2 Characteristics of patients by gender and age

\begin{tabular}{|lccccccc|}
\hline $\begin{array}{l}\text { Parameters } \\
\text { Age (years) }\end{array}$ & Total & $\begin{array}{c}\text { Male } \\
\text { Diabetes }\end{array}$ & $\%$ & Total & $\begin{array}{c}\text { Female } \\
\text { Diabetes }\end{array}$ & $\%$ & $P$ \\
\hline $12-19$ & 180 & 2 & 1.1 & 228 & 6 & 2.6 & .2 \\
$20-29$ & 384 & 17 & 4.4 & 942 & 44 & 4.7 & .4 \\
$30-39$ & 456 & 65 & 14.3 & 761 & 82 & 10.8 & .04 \\
$40-49$ & 364 & 109 & 29.9 & 658 & 217 & 33 & .2 \\
$50-59$ & 322 & 194 & 60.2 & 637 & 364 & 57.1 & .2 \\
$60-69$ & 247 & 344 & 71.8 & 308 & 200 & 64.9 & .04 \\
$\geq 70$ & 204 & 134 & 65.7 & 180 & 111 & 61.7 & .2 \\
\cline { 1 - 2 } Total & 2254 & 768 & 34.1 & 3714 & 1024 & 27.6 & $<.0001$ \\
\hline \multicolumn{2}{l}{ Data are number (\%). } & & & & & & \\
\hline
\end{tabular}

Among patients with diabetes, the prevalence of body mass index (BMI) of $\geq 25$ was $85.7 \%(P<.0001)$, with a higher prevalence of BMI of $\geq 25$ in females $(87.7 \%)$ as compared to males (83.1\%) $(P=.008)$. The prevalence of diabetes in relation to BMI in the groups is shown in (Figure 1). The distribution of BMI according to gender among patients with diabetes is shown in (Table 3). ${ }^{8}$ 
Table 3 Characteristics of patients stratified by body mass index and gender

\begin{tabular}{|lcccccccc|}
\hline $\begin{array}{l}\text { Parameters } \\
\begin{array}{l}\text { Body mass } \\
\text { index }\left(\mathrm{Kg} / \mathrm{m}^{2}\right)\end{array}\end{array}$ & Total & Diabetes & $\%$ & Total & Diabetes & $\%$ & $P$ \\
\hline$<18.5$ & 81 & 2 & 2.5 & 155 & 17 & 11.0 & .02 \\
$18.5-24.9$ & 492 & 120 & 24.4 & 853 & 101 & 11.8 & $<.0001$ \\
$25-29.9$ & 803 & 281 & 35 & 946 & 231 & 24.4 & $<.0001$ \\
$30-34.9$ & 568 & 222 & 39.1 & 899 & 300 & 33.4 & .02 \\
$35-39.9$ & 193 & 76 & 39.4 & 506 & 204 & 40.3 & .4 \\
$\geq 40$ & 49 & 21 & 42.9 & 217 & 106 & 48.8 & .3 \\
\hline Data are number (\%). & & & & & & & & \\
\hline
\end{tabular}

Another study of (Obesity and Associated Factors - Kingdom of Saudi Arabia, 2013) from April through June 2013, where a total of 12,000 households, contacted, and 10,735 participants completed the survey (response rate 89.4\%).

Compared with male respondents, female respondents were younger, more likely to be married or previously married, less educated, and overweight or obese. Overall, 28.7\%, or 3.6 million, Saudis aged 15 years or older were obese.

The prevalencewas ranged from $24.1 \%$ among men to $33.5 \%$ among women. Both men and women consumed low amounts of fruits and vegetables (more than $81.0 \%$ of men and women consumed fewer than 3 servings of fruits and vegetables per day) and most were physically inactive ( $46.0 \%$ of men, and $75.1 \%$ of women practiced low to no physical activity at all).Risk of obesity was lower among men who reported high levels of physical activity than among inactive men. Men who were previously married, consumed 3 or more servings of meat per day, those who were previously diagnosed with diabetes or hypercholesterolemia, and those who had elevated blood pressure were more likely to be obese.

Among women, risk of obesity increased with age, being married or previously married compared with those never married, having been diagnosed with a chronic condition, and being pre-hypertensive or hypertensive. Women who had more education than high school were less likely to be obese than those who had a primary school educational level or less. Diet indicators and physical activity were not significantly associated with obesity among women. ${ }^{9}$

In the study of hypertensive and diabetic patients attending the primary health care clinics in Riyadh, only $19 \%$ of patients were found to have ideal weight (BMI 25 > ,kg/m2), while 35\% were overweight (BMI, 25-29.9 kg/m2), 41\% were moderately obese (BMI, 30-40 kg/m2) and 5\% were morbidly obese (BMI $40<\mathrm{kg} / \mathrm{m} 2$ ).

Another study showed that of the males studied, $20.7 \%$ were obese (BMI, $25-29.9 \mathrm{~kg} / \mathrm{m} 2$ ) and $37 \%$ were overweight (BMI $30<\mathrm{kg} / \mathrm{m} 2$ ); and among the females, $39.3 \%$ were obese and $27 \%$ were overweight. Our logistic regression results showed that BMI 25 <and age, but not gender, were significantly associated with diabetes $(\mathrm{P}=.01, \mathrm{P} \leq .0001$ and 0.2 , respectively).Both obesity and diabetes are preventable. Previous studies have demonstrated that changes in lifestyle are effective in preventing both diabetes and obesity in high-risk adults with impaired glucose tolerance. Increasing physical activity, improving diet and then sustaining these lifestyle changes can reduce both body weight and risk of diabetes. Health professionals must continue to stress the importance of a balanced diet and physical activity for healthy weight loss. In the Saudi society, men and women must overcome many obstacles to make the best choices for optimal health.

\section{Discussion}

Diabetes and obesity are multi factorial diseases of considerable heterogeneity. The prevalence of diabetes worldwide will see an increase of $42 \%$ between the years 2003 and 2025. Reported prevalence data from the Gulf region revealed high rates in Bahrain (25.7\%) and Oman (16.1\%). Epidemiological studies of the prevalence of diabetes in Saudi Arabia, 1982-2009. 
Globally, diabetes prevalence is similar in males and females, but it is slightly higher in men60>years of age and in females at older ages, which was not observed in our review. Overall, diabetes prevalence is higher in males, but there are more females than males with diabetes, which was true in thereview for females in the younger age groups. The dramatic increase in the prevalence of diabetes can be explained by several factors. First, it is a disorder of the elderly. Over $20 \%$ of individuals aged 65 or more have diabetes. Studies from Saudi Arabia have shown different age-specific prevalence rates. Our data demonstrate an increasing prevalence of diabetes mellitus with advancing age; the fact that diabetes prevalence increases with age is consistent with the findings in previous studies. Second, diabetes is closely linked to obesity.

The provision of clinical preventive services to identify and control hypertension, elevated cholesterol levels, obesity and diabetes remain important medical priorities nationally. Development and implementation of national programs to promote a balanced diet, increased physical activity and weight control must be national priorities as well.

The results of the review studies have three important implications for national diabetes prevention and diabetes management programs. First, it appears that diabetes prevalence rates will almost certainly continue to rise in the Saudi population over the next two decades. The rapid aging of the currently very young Saudi population to a high-risk older age, coupled with emerging life-prolonging diabetes treatments, will maintain a balance between incidence and mortality in the foreseeable future. Even if incidence rates were to flatten out or decline due to a breakthrough in diabetes prevention, prevalence rates would continue to rise as incidence outpaces mortality. As a result, the health burden due to all types of diabetic complications will likely continue.

This means that the health care and social service systems should start preparing now to provide the secondary prevention and support services and systems that a large number of adults with diabetes are going to require to maintain a reasonably good quality of life. These include diabetes-screening programs, foot-care programs, accessible dialysis services, dietary counseling services and an enhanced infrastructure at the community level to facilitate independent living by adults with limited mobility and eyesight. Second“ ,upstream "population-based primary prevention programs need to be aggressively implemented to ensure that diabetes incidence begins to decrease in the future.

The dramatically higher rates of diabetes in the Saudi population highlight the urgency of this activity. Because diabetes appears to be closely related to the adoption of many aspects of the modern lifestyle, including diet and low levels of physical activity, prevention programs that draw upon traditional practices need to be implemented.A number of very promising primary prevention programs that draw upon older traditions and ways of life have been implemented in our institution. Third, the reason for the higher prevalence of diabetes in Saudi women below 50 years of age observed in this study also needs to be better understood. The relationship to earlier episodes of gestational diabetes should be investigated as one possibility.

\section{Conclusion}

This review concluded that diabesity has already become a worldwide epidemic with a significant health and economic burden affecting both developed and developing countries. Numerous studies have examined the situation; however, further data are still needed to evaluate the economic burden, particularly of obesity, in developing countries in different parts of the world including the Middle East, Africa, parts of Asia and parts of South America, where data are still scarce. Research studies are highly encouraged in developing countries in order to identify the epidemiology of diabesity, its economic and health burden and its associated risk factors. ${ }^{10}$

Diabetes has a complex pathophysiology and management strategy. Obesityis the main risk factor attributed to diabetes in the Arab world. The sedentary lifestyle in most of Arab countries, coupled with a hot climate and unique cultural barriers to physical activities, are important factors to be considered in the rapid increase in the prevalence of obesity among Arab countries. Most of the available epidemiological data show that Arab females are more prone to obesity and diabetes. Several factors could account for these findings, but especially important are the persistent social and cultural barriers to physical activities.

The unusual age-related disease has been highlighted in several epidemiological studies of the prevalence of obesity in Arab schoolchildren. The widespread epidemic of obesity-related diabetes in Arab populations has been analyzed in a limited number of genetic studies. 
Finally, there is a lack of quality data regarding obesity-related diabetes in Arab populations. Gaps exist in the information about diabetes and obesity, in particular in relation to ethnic specific cut-off points for diagnosis and treatment of diabetes. In addition, further GWAS in obese and diabetic Arab populations could add to our understanding of the path physiology, prevention and reversal of this disease. ${ }^{11}$

\section{Recommendations}

- $\quad$ Extensive health education and promotion activities on obesity relations with diabetes especially most at risk groups and prevention measures.

- $\quad$ Expand research on heterogeneity.

- $\quad$ Emphasize primary prevention of obesity and type 2 diabetes.

- Develop innovative approaches to pharmacological and surgical management.

- $\quad$ Adopt a chronic disease model linking obesity to diabetes care.

- $\quad$ Screening for Pre-Diabetes and Diabetes

Testing should be considered in all adults who are overweight (BMI > $25 \mathrm{~kg} / \mathrm{m} 2$ ) and have additional risk factors:

- $\quad$ Physical inactivity

- $\quad$ First-degree relative with diabetes

- Members of a high-risk ethnic population (e.g., African American, Latino, Native American, Asian American, Pacific Islander)

- Women who delivered a baby weighing $>4 \mathrm{~kg}$ or were diagnosed with gestational diabetes mellitus

- Hypertension (>140/90 mmHg or on therapy for hypertension)

- $\quad$ other clinical conditions associated with insulin resistance (e.g., severe obesity, acanthusesNigerians polycystic ova rain syndrome)

- $\quad$ History of cardiovascular disease. ${ }^{11}$

\section{Compliance with ethical standards}

\section{Acknowledgments}

We are grateful to all those who assist us in the production of this review, special thanks to Professor Sufian Khalid.

\section{Disclosure of conflict of interest}

The authors declare that they have no conflict of interest.

\section{References}

[1] Diabetes. June 2017 Accessed January 28, 2018.

[2] International Diabetes Federation. Diabetes Atlas. 5th ed. IDF Publications; Brussels, Belgium. The Global Burden of Diabetes. 2011; 7-13.

[3] Aguiree Florencia, Brown Alex, Cho Nam Ho, Dahlquist Gisela, Dodd Sheree, Dunning Trisha, Hirst Michael, Hwang Christopher, Magliano Dianna, Patterson Chris, Scott Courtney, Shaw Jonathon, Soltesz Gyula, UsherSmith Juliet, Whiting David. IDF Diabetes Atlas: sixth edition. 6th ed. International Diabetes Federation; Basel, Switzerland. 2013.

[4] Al-Nozha MM, Al-Maatouq MA, Al-Mazrou YY, Al-Harthi SS, Arafah MR, Khalil MZ. Diabetes mellitus in Saudi Arabia. Saudi Med J. 2004 Nov;25(11): 1603-10.

[5] Alqurashi Khalid A, Aljabri Khalid S, Bokhari Samia A. Prevalence of diabetes mellitus in a Saudi community. Ann Saudi Med. 2011 Jan-Feb;31(1):19-23.

[6] Haffner S, H Taegtmeyer. Epidemic obesity and the metabolic syndrome. Circulation. 2003; 108(13): 1541-1545.

[7] Gallagher EJ, D LeRoith, E Karnieli. Insulin Resistance in Obesity as the Underlying Cause for the Metabolic Syndrome. Mount Sinai Journal of Medicine: A Journal of Translational and Personalized Medicine. 2010; 77(5): 511-523. 
[8] Alqurashi, Khalid A, Khalid S. Aljabri, Samia A. Bokhari. Prevalence of diabetes mellitus in a Saudi community. Annals of Saudi medicine. 2011; 31(1): 19.

[9] Memish, Ziad A., et al. Peer reviewed: Obesity and associated factors-Kingdom of Saudi Arabia, 2013. Preventing chronic disease. 2014; 11.

[10] Farag, Youssef MK, and Mahmoud R. Gaballa. "Diabesity: an overview of a rising epidemic." Nephrology Dialysis Transplantation. 2010; 26(1): 28-35.

[11] Abuyassin B, I Laher. Obesity-linked diabetes in the Arab world: a review. Eastern Mediterranean Health Journal. $2015 ; 21(6): 420$. 